\title{
Towards Affect-sensitive Assistive Intervention Technologies for Children with Autism
}

\author{
Karla Conn, Changchun Liu, Nilanjan Sarkar, \\ Wendy Stone and Zachary Warren \\ Vanderbilt University \\ USA
}

\section{Introduction}

Investigation into technology-assisted intervention for children with autism spectrum disorder (ASD) has gained momentum in recent years. Therapists involved in interventions must overcome the communication impairments generally exhibited by children with ASD by adeptly inferring the affective cues of the children to adjust the intervention accordingly. Similarly, an intelligent system, such as a computer or robot, must also be able to understand the affective needs of these children - an ability that the current technologyassisted ASD intervention systems lack - to achieve effective interaction that addresses the role of affective states in human-computer interaction (HCI), human-robot interaction (HRI), and intervention practice. In this chapter we present a physiology-based affect-inference mechanism for emotion modeling, emotion recognition, and emotion-sensitive adaptive response in technology-assisted intervention. This work is the first step towards developing "understanding" interactive technologies for use in future ASD intervention. We address the problem of how to make computer-based ASD intervention tools affect-sensitive by designing therapist-like affective models of the children with ASD based on their physiological responses. By employing these models, we explain how a robot can detect the affective states of a child with ASD and adapt its behaviors accordingly. Experimental results with 6 children with ASD from computer-based cognitive tasks and a proof-ofconcept experiment (i.e., a robot-based basketball game) are presented. A Support Vector Machines (SVM) based affective model yielded approximately $82.9 \%$ success for predicting affect inferred from a therapist. The robot learned the individual liking level of each child with regard to the game configuration and selected appropriate behaviors to present the task at his/her preferred liking level. Results show the robot automatically predicted individual liking level in real time with $81.1 \%$ accuracy. This is the first time, to our knowledge, that the affective states of children with ASD have been detected via a physiology-based affect recognition technique in real time. This is also the first time that the impact of affect-sensitive closed-loop interaction between a robot and a child with ASD has been demonstrated experimentally.

While there is at present no single accepted intervention, treatment, or known cure for ASD, there is growing consensus that intensive behavioral and educational intervention programs can significantly improve long term outcomes for individuals and their families (Rogers, 
1998). Despite the urgent need and societal import of intensive treatment (Rutter, 2006), appropriate intervention resources for children with ASD and their families are often extremely costly when accessible (Jacobson et al., 1998; Tarkan, 2002). Therefore, an important new direction for research on ASD is the identification and development of assistive intervention tools that can make application of intensive treatment more readily accessible.

In response to this need, a growing number of studies have been investigating the application of advanced interactive technologies to address core deficits related to autism, namely computer technology (Bernard-Opitz et al., 2001), virtual reality environments (Pares et al., 2005; Parsons \& Mitchell, 2002), and robotic systems (Dautenhahn \& Werry, 2004; Kozima et al, 2005; Michaud \& Theberge-Turmel, 2002; Pioggia et al., 2005; Scassellati, 2005). Initial results indicate that such technologies may hold promise for rehabilitation of children with ASD. Computer and virtual reality (VR) based intervention may provide a simplified but exploratory interaction environment for children with ASD (Moore et al., 2000; Parsons \& Mitchell, 2002). Various software packages and VR environments have been developed and applied to address specific deficits associated with autism, e.g., understanding of false belief (Swettenham, 1996), attention (Trepagnier et al., 2006), expression recognition (Silver \& Oakes, 2001), and social communication (Bernard-Opitz et al., 2001; Parsons et al., 2005). Research suggested that robots can allow simplified but embodied social interaction that is less intimidating or confusing for children with ASD (Dautenhahn \& Werry, 2004). Michaud \& Theberge-Turmel (2002) investigated the impact of robot design on the interactions with children and emphasized that systems need to be versatile enough to adapt to the varying needs of different children. Pioggia et al. (2005) developed an interactive life-like facial display system for enhancing emotion recognition in individuals with ASD. Robots have also been used to teach basic social interaction skills using turn-taking and imitation games, and the use of robots as social mediators and as objects of shared attention can encourage interaction with peers and adults (Dautenhahn \& Werry, 2004; Kozima et al, 2005). Interactive technologies pose the advantage of furnishing robust systems that can support multimodal interaction and provide a repeatable, standardized stimulus while quantitatively recording and monitoring the performance progress of the children with ASD to assess the intervention approaches (Scassellati, 2005). By employing human-computer interaction (HCI) and human-robot interaction (HRI) technologies, interactive therapeutic tools can partially automate the time-consuming, routine behavioral therapy sessions and may allow intensive intervention to be conducted at home (Dautenhahn \& Werry, 2004). For the purpose of using our affective computing tools, computers or robots could be the mode of technology for assisted ASD interventions. We will use the term intelligent system primarily in this text to imply both computer and robot interactive technologies.

Even though there is increasing research in assistive technologies for autism intervention, the authors found no published studies that specifically addressed how to automatically detect and respond to affective cues of children with ASD. This could be important since research suggests that people tend to interact with computers as they might relate to other people, provided that the technology behaves in a socially competent manner (Reeves \& Nass, 1996). We believe that such ability could be critical given the importance of human affective information in HRI (Fong et al., 2003; Picard, 1997) and the significant impacts of the affective factors of children with ASD on the intervention practice (Seip, 1996). Common 
in autism intervention, therapists who work with children with ASD continuously monitor affective cues of the children in order to make appropriate decisions about adaptations to their intervention strategies. For example, 'likes and dislikes chart' is recommended to record the children's preferred activities and/or sensory stimuli during interventions that could be used as reinforcers and/or 'alternative behaviors' (Seip, 1996). Children with autism are particularly vulnerable to anxiety and intolerant of feelings of frustration, which requires a therapist to plan tasks at an appropriate level of difficulty (Ernsperger, 2003). The engagement of children with ASD is the ground basis for the 'floor-time therapy' to help them develop relationships and improve their social skills (Wieder \& Greenspan, 2005).

The potential impacts brought by an intelligent system that can detect the affective states of a child with ASD and interact with him/her based on such perception could be various. Complex social stimuli, sophisticated interactions, and unpredictable situations could be gradually but automatically introduced when the robot recognizes that the child is comfortable or not anxious at a certain level of interaction dynamics for a reasonably long period of time. A therapist could use the child's affective records to analyze the therapeutic approach. With the record of the activities and the consequent emotional changes in a child, an intelligent system could learn individual affective characteristics over time and thus could adapt the ways it responds to the needs of different children.

The primary objective of the current research is to investigate how to augment HCI and HRI to be used in autism intervention by endowing the intelligent system with the ability to recognize and respond to the affective states of a child with ASD. To achieve this objective, the research is divided into two phases. Phase I represents the development of affective models through psychophysiological analysis, which includes designing cognitive tasks for affect-elicitation, deriving physiological features via signal processing, and developing affective models using machine learning techniques. Phase II is characterized by the investigation of affect sensitivity during closed-loop interaction between a child with ASD and the intelligent system (i.e., computer, VR environment, or robot). A proof-of-concept experiment was designed wherein a robot learns individual preferences based on the predicted liking level of the children with ASD and in real time selects an appropriate behavior accordingly.

The chapter is organized as follows: The scope and rationale of this work is presented in Section 2. Section 3 describes our use of physiological indices for affect recognition and our proposed framework for automatically detecting and responding to affective cues of children with ASD in closed-loop interaction, as well as the experimental design. This description is followed by a detailed results and discussion section (Section 4). Finally, Section 5 summarizes the contributions of the paper and outlines possible future directions of this research. In addition, the machine learning algorithms employed in this study is presented in the Appendix.

\section{Scope and rationale}

The overview of the affect-sensitive closed-loop interaction between a child with ASD and an intelligent system is presented in Fig. 1. The physiological signals from the children with ASD are recorded when they are interacting with the system. These signals are processed in real time to extract features, which are fed as input into the models developed in Phase I. The models determine the perceived affective cues and return this information as an output. 
The affective information, along with other environmental inputs, is used by a controller to decide the next course of action for the intelligent system. The child who engages with the system is then influenced by the system's behavior, and the closed-loop interaction cycle begins anew. The impact of such an interaction with a robot as the intelligent system is evaluated in Phase II.

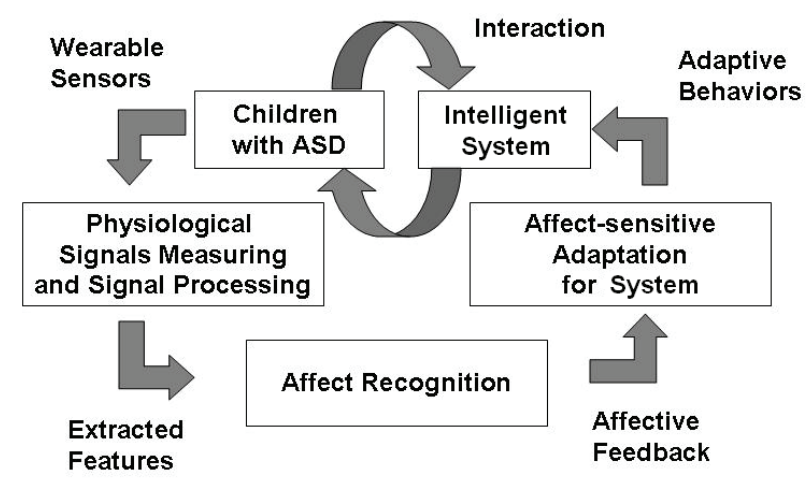

Fig. 1. Framework overview

Human interactions with technology are characterized by explicit as well as implicit channels of communication with presumed underlying affective states (Picard, 1997). While the explicit channel transmits overt messages, the implicit one transmits hidden messages about the communicator (e.g., his/her intention and attitude). There is a growing consensus that endowing an intelligent system with an ability to understand implicit affective cues should permit more meaningful and natural HCI and HRI (Picard, 1997). There are several modalities such as facial expression (Bartlett et al., 2003), vocal intonation (Lee \& Narayanan, 2005), gestures and postures (Asha et al., 2005; Kleinsmith et al., 2005), and physiology (Kulic \& Croft, 2007; Liu et al., 2006; Mandryk \& Atkins, 2007; Picard et al., 2001; Rani et al., 2004) that can be utilized to evaluate the affective states. In this work we chose to create affective models based on physiological data for several reasons. Children with ASD often have communicative impairments (both nonverbal and verbal), particularly regarding expression of affective states (DSM-IV-TR, American Psychiatric Association, 2000; Green et al., 2002; Schultz, 2005). These vulnerabilities place limits on traditional conversational and observational methodologies; however, physiological signals are continuously available and are not necessarily directly impacted by these difficulties (Ben Shalom et al., 2006; Groden et al., 2005; Toichi \& Kamio, 2003). As such, physiological modeling may represent a methodology for gathering rich data despite the potential communicative impairments of children with ASD. In addition, physiological data may offer an avenue for recognizing aspects of affect that may be less obvious for humans but more suitable for computers by using signal processing and pattern recognition tools. Furthermore, there is evidence that the transition from one affective state to another state is accompanied by dynamic shifts in indicators of Autonomic Nervous System (ANS) activity (Bradley, 2000). The physiological signals that have been used in this research consist of various cardiovascular, electrodermal, 
electromyographic, and body temperature signals, all of which have been extensively investigated in psychophysiology literature (Bradley, 2000).

An important question when estimating human affective response is how to operationalize the affective states. Although much existing research on affective modeling categorizes affective states into "basic emotions," there is no consensus on a set of basic emotions among the researchers (Cowie et al., 2001). This fact implies that pragmatic choices are required to select target affective states for a given application (Cowie et al., 2001). In this research we chose anxiety, engagement, and liking to be the target affective states. Anxiety was chosen for two primary reasons. First, anxiety plays an important role in various human-machine interaction tasks that can be related to task performance (Brown et al., 1997). Second, anxiety frequently co-occurs with ASD and plays an important role in the behavior difficulties of children with autism (Gillott et al., 2001). Engagement, defined as "sustained attention to an activity or person," has been regarded as one of the key factors for children with ASD to make substantial gains in academic, communication, and social domains (Ruble \& Robson, 2006). With 'playful' activities during the intervention, the liking of the children (i.e., the enjoyment they experience when interacting with an intelligent system) may create the urge to explore and allow prolonged interaction for the children with ASD, who are susceptible to being withdrawn (Dautenhahn \& Werry, 2004).

Notably, there is evidence that several affective states could co-occur at different arousal levels (Vansteelandt et al., 2005), and different individuals could express the same emotion with different characteristic response patterns under the same contexts (i.e., phenomenon of person stereotypy) (Lacey \& Lacey, 1958). The novelty of the presented affective modeling is that it is individual-specific to accommodate the differences encountered in emotional expression, and it consists of an array of recognizers - each of which determines the intensity of one target affective state for each individual. In this work, a therapist observed the experiments (described in Section 3.2.2) and provided subjective reports based on expertise in inferring presumable underlying affective states from the observable behaviors of children with ASD. The therapist's reports on perceived intensity of the affective states of a child and the extracted physiological indices (described in Section 3.2.4) were employed to develop therapist-like affect recognizers that predict high/low levels of anxiety, engagement, and liking for each child with ASD.

Once affective modeling was completed in Phase I, the recognizers equipped the intelligent system with the capability to detect the affective states of the children with ASD in real time from on-line extracted physiological features, which could be utilized in future interventions even when a therapist is not available. As stated in (Dautenhahn et al., 2003), it is important to have robots maintain characteristics of adaptability when applied to autism intervention. In Phase II, we designed and implemented a proof-of-concept experiment (robot-based basketball) wherein a robot adapts its behaviors in real time according to the preference of a child with ASD, inferred from the interaction experience and the predicted consequent liking level. This work is the first time, to our knowledge, that the feasibility and the impact of affect-sensitive closed-loop interaction between a robot and a child with ASD have been demonstrated experimentally. While the results are achieved in a non-social interaction task, it is expected that the real-time affect recognition and response system described in this work will provide a basis for future research into developing technology-assisted intervention tools to help children with ASD explore social interaction dynamics in an affect-sensitive and adaptive manner. 


\section{Experimental investigation}

\subsection{Participants}

Given the nature of autism (a spectrum disorder) which implies vast individual differences, the works on autism intervention assistive tools are generally guided by the individual characteristics, needs, and preferences of the children (i.e., individual-specific approach) and focus on one sect of the population to develop a method with the flexibility to make future modifications for a wider part of the population (Pioggia et al., 2005; Robins et al., 2005; Robins et al., 2004; Werry et al., 2001). The spectrum nature of autism and the phenomenon of person stereotypy (Lacey \& Lacey, 1958) led us to choose an individual-specific approach to work on a long-term basis with a small group of children with ASD in order to evaluate our affect-sensitive intelligent system.

Six participants within the age range of 13 to 16 years old volunteered to partake in the experiments with the consent of their parents. Each of the participants had a diagnosis on the autism spectrum, either autistic disorder, Asperger's Syndrome, or pervasive developmental disorder not otherwise specified (PDD-NOS), according to their medical records. Due to the nature of the designed cognitive tasks (as described in Section 3.2.1), the following were considered when choosing the participants: (i) having a minimum competency level of age-appropriate language and cognitive skills and (ii) not having any history of mental retardation. Each child with ASD underwent the Peabody Picture Vocabulary Test III (PPVT-III) (Dunn \& Dunn, 1997) to assess cognitive function. The PPVTIII is a measure of single-word receptive vocabulary that is often used as a proxy for intelligence quotient (IQ) testing (Dunn \& Dunn, 1997). It provides standard scores with a mean of 100 and a standard deviation of 15. The PPVT-III measure has high correlations with standardized tests such as the Stanford-Binet Intelligence Scale and the Wechsler Intelligence Scale for Children (Bee \& Boyd, 2004), and DSM-IV-TR (2000) classifies full scale IQ's above 70 as nonretarded. Inclusion in our study was characterized as obtaining a standard score of 80 or above on the PPVT-III measure. Table 1 shows the characteristics of the participants in the experiments. The group sizes and the cardinality of participant age range of many studies on technology-assisted autism intervention are commensurate with our work when an individual-specific approach was used (Pioggia et al., 2005; Robins et al., 2005; Robins et al., 2004; Werry et al., 2001). The affective modeling was performed based on a large sample size of observations (approximately 85 epochs over 6 hours) for each child with ASD, which is comparatively more extensive than many other works (Groden et al., 2005; Pioggia et al., 2005; Robins et al., 2004).

\begin{tabular}{|c|c|c|c|c|}
\hline Child ID & Gender & Age & Diagnosis & PPVT-III Score \\
\hline A & Male & 15 & Autistic Disorder & 99 \\
\hline B & Male & 15 & Asperger's Syndrome & 80 \\
\hline C & Male & 13 & Autistic Disorder & 81 \\
\hline D & Male & 14 & PDD-NOS & 92 \\
\hline E & Male & 16 & PDD-NOS & 93 \\
\hline F & Female & 14 & PDD-NOS & 83 \\
\hline
\end{tabular}

Table 1. Characteristics of Participants. 


\subsection{Phase I - affective modeling}

While the impact of Phase II is evaluated on affect-sensitive human-robot interaction, we built the affective models using physiological data gathered from two human-computer interaction tasks. Our previous work (Rani et al., 2006a) showed that affective models built through human-computer interaction tasks could be successfully employed to achieve affect recognition in human-robot interaction for typical individuals. This observation suggests that it is possible to broaden the domain of tasks for affective modeling, thus reducing the habituation effect of continuous exposure to the same robotic system.

\subsubsection{Task design for affect elicitation during cognitive tasks}

Two computer-based cognitive tasks - an anagram-solving task and a Pong-playing task were designed to evoke varying intensities of the following three affective states: anxiety, engagement, and liking, from the participants. Affective responses were manipulated by presenting the participant with anagrams of varying difficulty levels. For example, a long series of trivially easy anagrams caused less engagement. The Pong task involved the participant playing a variant of the classic video game "Pong." Various parameters of the game were manipulated to elicit the required affective responses: ball speed and size, paddle speed and size, sluggish or over-responsive keyboard, and the level of the computer opponent player. For examples, very high speeds and sluggish or over-responsive keyboard caused anxiety at times and playing against a moderate-level computer player usually generated liking. The task configurations were established through pilot work.

Each task sequence was subdivided into a series of discrete trials/epochs that were bounded by the subjective affective state assessments. These assessments were collected using a battery of five questions regarding the three target affective states and the perceived difficulty and performance rated on an eight-point Likert scale where 1 indicated the lowest level and 8 indicated the maximum level. Each participant took part in six sessions - three one-hour sessions of anagrams and three one-hour sessions of Pong - on six different days.

\subsubsection{Experimental setup}

Fig. 2 shows the setup for the experiment. A child with ASD was involved in the cognitive tasks on computer $\mathrm{C} 1$ while his/her physiological data was acquired via wearable biofeedback sensors and the Biopac system (www.biopac.com). After being amplified and digitized, physiological signals were transferred from the Biopac transducers to C2 through an Ethernet link and stored. C1 was also connected to the Biopac system via a parallel port, through which the physiological data were recorded in a time-synchronized manner. To gain perspective from different sources and enhance the reliability of the subjective report, a therapist with experience in working with children with ASD and a parent of the participant were also involved in the study, who may best know the participant. We video recorded the sessions to cross-reference observations made during the experiment. The signal from the video camera was routed to a television, and the signal from the participant's computer screen where the task was presented was routed to a separate computer monitor M2. The therapist and a parent were seated at the back of the experiment room, watching the experiment from the view of the video camera and observing how the task progressed on the separate monitor. 


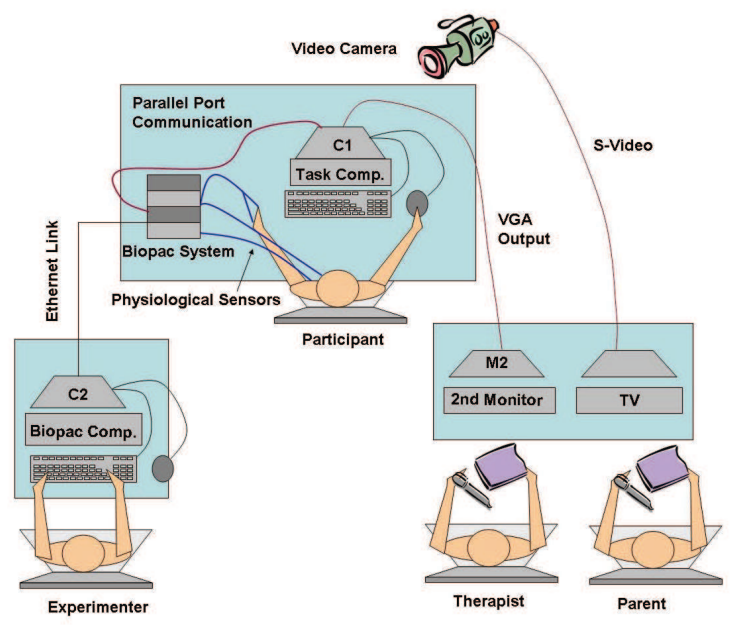

Fig. 2. Experimental setup for affective modeling tasks

\subsubsection{Experimental procedure}

On the first visit, participants completed the PPVT-III measurement to determine eligibility for the experiments. After initial briefing regarding the tasks, physiological sensors from a Biopac system were attached to the participant's body. Participants were asked to relax in a seated position and read age-appropriate leisure material while a three-minute baseline recording was performed, which was later used to offset day-variability. Each session lasted about an hour and consisted of a set (13-15) of either 3-minute epochs for anagram tasks or up to 4-minute epochs for Pong tasks. Each epoch was followed by subjective report questions rated on an eight-point Likert scale. The three sets of reports were used as the possible reference points to link the objective physiological measures to the participant's affective state.

\subsubsection{Physiological indices for affective modeling}

There is good evidence that the physiological activity associated with affective states can be differentiated and systematically organized (Bradley, 2000). Cardiovascular and electromyogram activities have been used to examine positive and negative affective states of people (Cacioppo et al., 2000; Papillo \& Shapiro, 1990). Electrodermal activities have been shown to be associated with task engagement (Pecchinenda \& Smith, 1996). The variation of peripheral temperature due to emotional stimuli was studied by Kataoka et al. (1998). In this work, we exploited the dependence of physiological responses on underlying affective states to develop affective models for children with ASD by using the machine learning method as described in Section 3.2.5 and Appendix 1. The physiological signals we examined were: various cardiovascular activities including electrocardiogram (ECG), impedance cardiogram (ICG), photoplethysmogram (PPG), and phonocardiogram (PCG)/heart sound; electrodermal activities (EDA) including tonic and phasic responses from skin conductance; electromyogram (EMG) activities from corrugator supercilii, zygomaticus major, and upper trapezius muscles; and peripheral temperature. These signals 
were selected because they are likely to demonstrate variability as a function of the targeted affective states, as well as they can be measured non-invasively, and are relatively resistant to movement artifacts (Lacey \& Lacey, 1958; Dawson et al., 1990). Further details of the physiological signals examined in this work along with the features derived from each signal can be found in our supplementary publication Rani et al. (2006b).

The physiological signals were acquired using the Biopac MP150 data acquisition system (www.biopac.com). ECG was measured from the chest using the standard two-electrode configuration. ICG describes the changes of thorax impedance due to cardiac contractility and was measured by four pairs of surface electrodes that were longitudinally configured on both sides of the body. A microphone specially designed to detect heart sound waves was placed on the chest to measure PCG. PPG, peripheral temperature, and EDA were measured from the middle finger, the thumb, and the index and ring fingers of the nondominant hand, respectively. EMG was measured by placing surface electrodes on two facial muscles (corrugator supercilii and zygomaticus major) and an upper back muscle (upper trapezius). Fig. 3 shows the sensor setup. The sampling rate was fixed at $1000 \mathrm{~Hz}$ for all the channels. Appropriate amplification and band-pass filtering were performed. Subsequently, emotional stimulus induced by cognitive tasks was applied in epochs of up to four minutes in length (as described in Section 3.2.1).
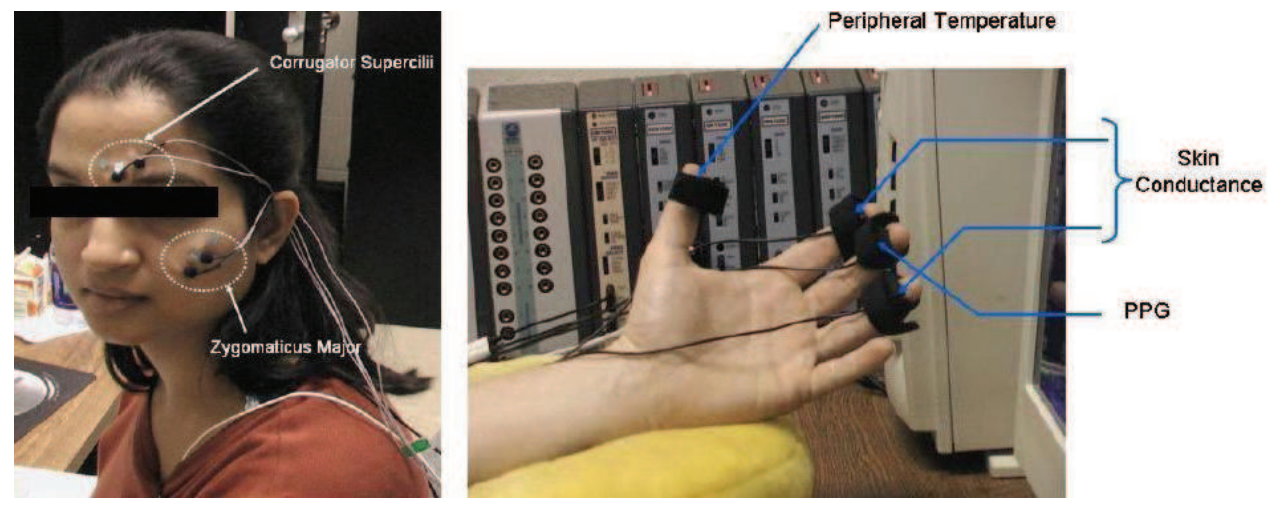

Fig. 3. Sensor Setup. (a) shows the position of facial EMG sensors and (b) shows the placement of sensors on non-dominant hand.

Signal processing techniques such as Fourier transform, wavelet transform, thresholding, and peak detection were used to derive the relevant features from the physiological signals. For example, inter beat interval (IBI) is the time interval between two " $R$ " waves in the electrocardiogram (ECG) waveform. Power spectral analysis is performed on the IBI data to localize the sympathetic and parasympathetic nervous system activities associated with two frequency bands (i.e., high $(0.15-0.4 \mathrm{~Hz})$ and low $(0.04-0.15 \mathrm{~Hz})$ frequency components. Photoplethysmograph (PPG) signal measures changes in the volume of blood in the finger tip associated with the pulse cycle and provides an index of the relative constriction versus dilation of the blood vessels in the periphery. Pulse transit time (PTT) is estimated by computing the time between systole at the heart (as indicated by the R-wave of the ECG) and the peak of the pulse wave reaching the peripheral site where PPG is being measured. The features extracted from the heart sound signal consist of the mean and standard deviation of the 3rd, 4th, and 5th level coefficients of the Daubechies wavelet transform. 
Bioelectrical impedance analysis (BIA) measures the impedance or opposition to the flow of an electric current through the body fluids contained mainly in the lean and fat tissue. A common variable in recent psychophysiology research, pre-ejection period (PEP) is derived from ICG and ECG and is most heavily influenced by sympathetic innervation of the heart. EDA consists of two main components - Tonic response and Phasic response. Tonic skin conductance refers to the ongoing or the baseline level of skin conductance in the absence of any particular discrete environmental events. Phasic skin conductance refers to the event related changes that occur, caused by a momentary increase in skin conductance (resembling a peak). The EMG signal from Corrugator Supercilii muscle (eyebrow) captures a person's frown and detects the tension in that region. This EMG signal is also a valuable source of blink information and helps determine the blink rate. The EMG signal from the Zygomaticus Major muscle captures the muscle movements while smiling. Upper Trapezius muscle activity measures the tension in the shoulders, one of the most common sites in the body for developing stress. Variations in the peripheral temperature mainly come from localized changes in blood flow caused by vascular resistance or arterial blood pressure and reflect the autonomic nervous system activity.

\subsubsection{SVM-based affective modeling}

Determining the intensity (e.g., high/low) of a particular affective state from the physiological response resembles a classification problem where the attributes are the physiological features and the target function is the degree of arousal. Our earlier work (Rani et al., 2006b) compared the efficacy of several machine learning algorithms (KNN, Bayesian Network Technique, Regression Tree, and SVM) to recognize the affective states from the physiological signals of typical individuals and found that SVM gave the highest classification accuracy. In this work, SVM was employed to determine the underlying affective state of a child with ASD given a set of physiological features. Details of the theory and learning methods of SVM can be found in (Vapnik, 1998) and are briefly described in Appendix 1.

As illustrated in Fig. 4, each participant had a data set comprised of both the objective physiological features and corresponding subjective reports on arousal level of target affective states from the therapist, the parent, and the participant. The physiological features were extracted by using the approaches described in Section 3.2.4). The individual range per affective state from each reporter on the subjective reports was normalized to $[0,1]$ and then discretized such that $0-0.50$ was labeled as low level and 0.51-1 was labeled as high level. All three affective states were partitioned separately so that there were two levels for each affective state. Each data set contained approximately 85 epochs. The multiple subjective reports were analyzed, and one was chosen as the possible reference points to link the physiological measures to the participant's affective state. For example, a therapist-like affect recognizer can be developed when the therapist's reports are used. A SVM-based recognizer was trained on each individual's data set for each target affective state. In this work, in order to deal with the nonlinearly separable data, soft margin classifiers with slack variables were used to find a hyperplane with less restriction (Eqn. 1, Appendix 1) (Burges, 1998). RBF (Radial Basis Function) was selected as the kernel function because it often delivers better performance (Burges, 1998). A ten-fold cross-validation was used to determine the kernel parameter and regularization parameter (Eqn. 2, Appendix 1) of the recognizer. 


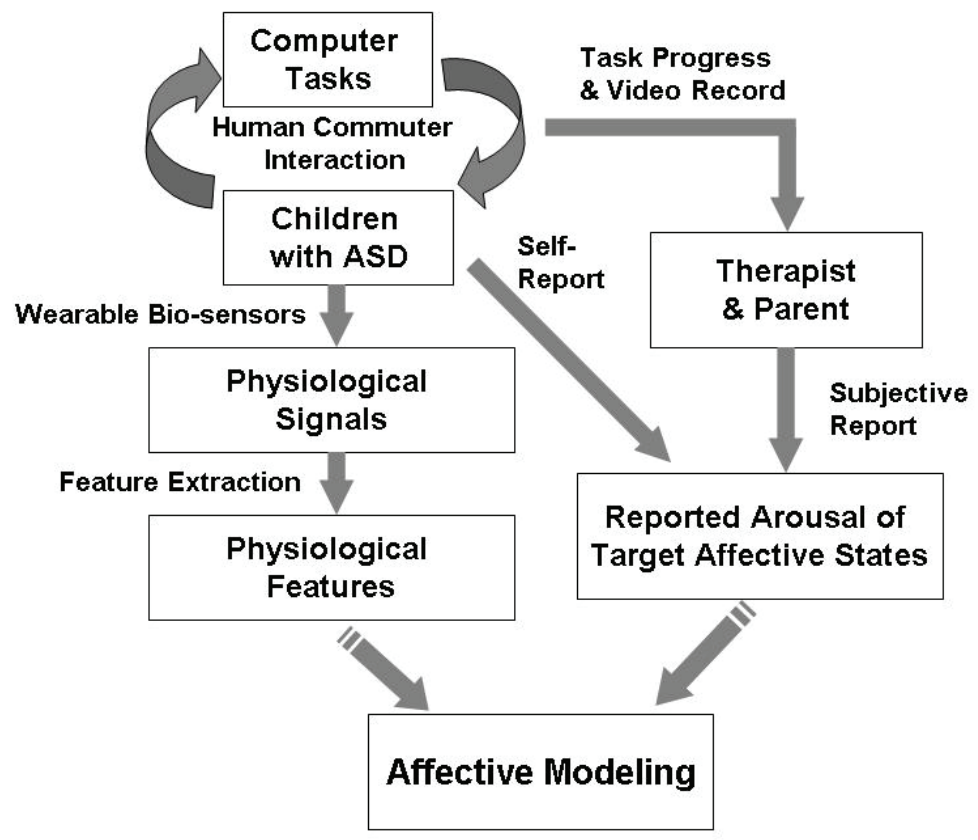

Fig. 4. Overview of affective modeling

Once affective modeling is accomplished, the affect recognizers can accept as input the physiological features extracted on-line and produce as output the probable level of the target affective state of a child with ASD while interacting with an intelligent system. In the design for the human-robot interaction task in Phase II, adequate measures were taken to avoid physical effort from overwhelming the physiological response.

\subsection{Phase II - closed-loop human robot interaction}

\subsubsection{Task design for affect-sensitive behavior adaptation task}

A closed-loop human robot interaction task, "robot-based basketball (RBB)," was designed. The main objective was two-fold: (i) to enable the robot to learn the preference of the children with ASD implicitly using physiology-based affective models as well as select appropriate behaviors accordingly; and (ii) to observe the effects of such affective-sensitivity in the closed-loop interaction between the children with ASD and the robot.

The affective model developed in Phase I is capable of predicting the intensity of liking, anxiety, and engagement simultaneously. However to designate a specific objective for the experiment in Phase II without compromising its proof-of-concept purpose, one of the three target affective states was chosen to be detected and responded to by the robot in real time. As has been emphasized in (Dautenhahn and Werry, 2004), the liking of the children (i.e., the enjoyment they experience when interacting with the robot) is a goal as desirable as skill learning for autism intervention. Therefore, liking was chosen as the affective state around which to modify the robot's behaviors in Phase II. 
In the RBB task, an undersized basketball hoop was attached to the end-effector of a robotic manipulator, which could move the hoop in different directions (as shown in Fig. 5) with different speeds. The children were instructed to shoot a required number of baskets into the moving hoop within a given time. Three robot behaviors were designed as shown in Table 2. For example, in behavior 1 the robot moves towards and away from the participant (i.e., in the $X$ direction) at a slow speed with soft background music, and the shooting requirement for successful baskets is relatively low. The parameter configurations were determined based on a pilot study to attain varied impacts on affective experience for different behaviors. From this pilot study, the averaged performance of participants for a given behavior was compiled and analyzed. Behavior transitions occurred between but not within epochs. As such, each robot behavior extended for the length of an epoch (1.5 minutes in duration) to have the participant fully exposed to the impact of that behavior.

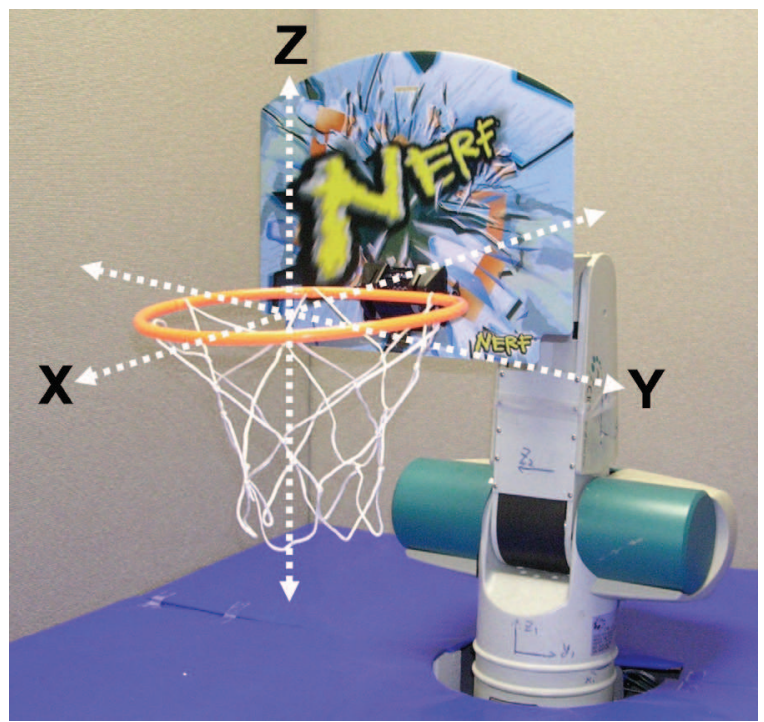

Fig. 5. $X, Y$, and $Z$ directions for behaviors used in RBB

\begin{tabular}{|c|c|c|c|c|}
\hline $\begin{array}{c}\text { Behavior } \\
\text { ID }\end{array}$ & $\begin{array}{c}\text { Motion } \\
\text { Direction }\end{array}$ & $\begin{array}{c}\text { Speed } \\
\text { (sec/period) }\end{array}$ & $\begin{array}{c}\text { Threshold } \\
\text { (shots/epoch) }\end{array}$ & $\begin{array}{c}\text { Background } \\
\text { Music }\end{array}$ \\
\hline 1 & $\mathrm{X}$ & 2 & 12 & Serene \\
\hline 2 & $\mathrm{Y}$ & 4 & 20 & Lively \\
\hline 3 & $\mathrm{Z}$ & 8 & 30 & Irregular \\
\hline
\end{tabular}

Table 2. Robot behaviors

Each of the six participants took part in two robot basketball sessions (RBB1 and RBB2). In RBB1 (non-affect based) the robot selected its behavior randomly (i.e., without any regard to the liking information of the participant), and the presentation of each type of behavior was evenly distributed. This session was designed for two purposes: (i) to explore the state space and action space of the QV-learning algorithm used in RBB2 for behavior adaptation (described in Section 3.3.4); and (ii) to validate that the different robot behaviors have 
distinguishable impact on the child's level of liking. In RBB2 (liking-based), the robot continues to learn the child's individual preference and selects the desirable behavior based on interaction experiences (i.e., records of robot behavior and the consequent liking level of a participant predicted by the affective model). The idea is to investigate whether the robot can automatically choose the most-liked behavior of each participant as observed from RBB1 by means of physiology-based affective model and QV-learning.

\subsubsection{Experimental setup}

The real-time implementation of the RBB system is shown in Fig. 6. The set-up included a 5 degrees-of-freedom robot manipulator (CRS Catalyst-5 System) Two infrared (IR) transmitter and receiver pairs were attached to the basketball hoop to detect small, soft foam balls going through the hoop. Biological feedback equipment (Biopac system) was connected to a $\mathrm{C} 1$ that: (i) acquired physiological signals from the Biopac system and extracted physiological features on-line, (ii) predicted the probable liking level by using the affective model developed in Phase I, (iii) acquired IR data through the analog input channels of the Biopac system, (iv) ran a QV-learning algorithm that learns the participant's preference and chooses the robot's next behavior accordingly. Computer $\mathrm{C} 1$ was connected serially to the CRS computer (C2), which ran Simulink software. The behavior switch triggers were transmitted from C1 to C2 via a RS232 link. The commands to control the robot's various joints were transmitted from C2 to the robot. As in Phase I tasks, the therapist and a parent were also involved, watching the experiment from the TV that was connected to a video camera.

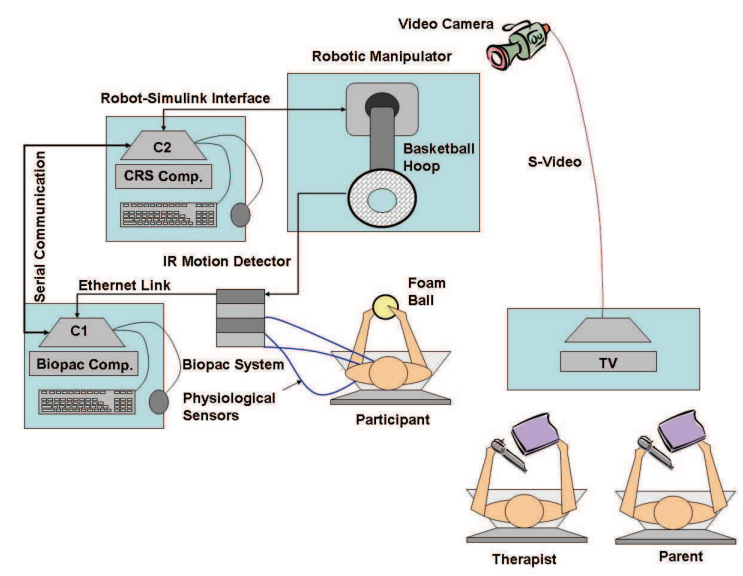

Fig. 6. Experimental set-up for robot basketball

\subsubsection{Experimental procedure}

Each basketball session (RBB1 or RBB2) was approximately 1 hour long and included 27 minutes of active human-robot interaction (i.e., 18 epochs of 1.5 minutes each). The remaining time was spent attaching sensors, guiding a short practice, taking a baseline recording, collecting subjective reports, and pausing for scheduled breaks. During the 
experiment, the participant was asked to take a break after every four epochs and the participant could request a break whenever he/she desired one. During each basketball epoch, the participant received commands and performance assessments from pre-recorded dialogue via a speech program running on $\mathrm{C} 1$ and the interaction proceeded as follows:

1. The participant was notified of the shooting requirement threshold.

2. A start command instructed the participant to start shooting baskets.

3. Once the epoch started, the participant was given voice feedback every 30 seconds regarding the number of baskets remaining and the time available.

4. A stop command instructed the participant to stop shooting baskets, which ended the epoch.

5. At the end of each epoch, the participant's performance was rated and relayed to him/her as excellent, above average, or below average.

Each epoch was followed by a subjective reporting procedure using the same protocol as Phase I that took 30-60 seconds to collect. After the subjective reports were complete, the next epoch would begin. To prevent habituation, a time interval of 7 days or more between RBB sessions was enforced.

\subsubsection{Affect-sensitive behavior adaptation in closed-loop human robot interaction}

We defined the state, action, state transition, and reward functions so that the affectsensitive robot behavior adaptation problem could be solved using the QV-learning algorithm as described in (Wiering, 2005) and Appendix 2.

The set of states consisted of three robot behaviors as described in Table 2. In every state, the robot has three possible actions $(1 / 2 / 3)$ that correspond to choosing behavior 1,2 , or 3 , respectively, for the next time step (i.e., next epoch). Each robot behavior persists for one full epoch and the state/behavior transition occurs only at the end of an epoch. The detection of consequent affective cues (i.e., the real-time prediction of the liking level for the next epoch) was used to evaluate the desirability of a certain action. A reward function was defined based on the predicted liking level. If the consequent liking level was recognized as high, the contributing action was interpreted as positive and a reward was granted $(r=1)$; otherwise the robot received a punishment $(r=-1)$. QV-learning uses this reward function to have the robot learn how to select the behavior that was expected to result in a high liking level and therefore positively influenced the actual affective (e.g., liking) experience of the child.

RBB1 enables state and action exploration through random, evenly distributed behaviorswitching actions. The V-function and Q-function are updated using Eqn. (3) and Eqn. (4) from Appendix 2. After RBB1, the subjective reports are analyzed to examine the impacts of different behaviors on each participant's preference. In RBB2 the robot starts from a nonpreferred behavior/state and continues the learning process by using Eqn. (3) and Eqn. (4). A greedy action selection mechanism is used to choose the behavior-switching action with the highest $Q$-value.

Because of the limited number of states and actions in this proof-of-concept experiment, tabular representation is used for the V-function and the Q-function. To prevent a certain action and/or state from being overly dominant and to counteract the habituation effect, the values of $Q(s, a)$ and $V(s)$ are bounded by using the reward or punishment encountered in the interaction. The parameters in Eqn. (3) and Eqn. (4) are chosen as $\alpha=0.8$ and $\gamma=0.9$. Before RBB1 begins, the initial values in the V-table and the Q-table are set to 0 . 


\section{Results and discussion}

In this section we present both the Phase I results of physiology-based affective modeling for children with ASD and Phase II results of the affect-sensitive closed-loop interaction between children with ASD and the robot.

\subsection{Phase I - affect detection}

One of the prime challenges of this work is attaining reliable subjective reports. Moreover, researchers are reluctant to trust the responses of adolescents on self-reports (Barkley, 1998). In order to overcome this difficulty, a therapist and a parent were involved by using the approaches described in the experimental setup. They observed the experiments and provided subjective reports based on their expertise/experience in inferring presumable underlying affective states from the observable behaviors of children with ASD.

To measure the amount of agreement among the different reporters, the kappa statistic was used (Siegel and Castellan, 1988). The kappa coefficient (K) measures pair-wise agreement among a set of reporters making category judgments, correcting for expected chance agreement. When agreement is complete, $K=1$; whereas, when there is only agreement as would be expected by chance, $K=0$. Fig. 7 shows results for $K$ averaged across the target affective states.



Fig. 7. Average Kappa Statistics between Reporters for Affective States

It was observed that the agreement between the therapist and parent $(\mathrm{T} / \mathrm{P})$ showed the largest $\mathrm{K}$ values (mean $=0.62)$ among the three possible pairs for each child $(p<0.05$, paired $\mathrm{t}$-test). When each child is examined individually, different trends arise, which revealed diverse affective characteristics of the children with ASD who partook in this study. The Kappa agreement between therapist and parent is substantial for Child A, Child B, Child D, and Child $\mathrm{F}$ and moderate for Child $\mathrm{C}$ and Child $\mathrm{E}$. Such results might stem from the fact that it could be difficult for the therapist or parent to distinguish certain emotion for a particular child with ASD. For example, the agreement between therapist and parent for the anxiety level of Child $\mathrm{C}$ and Child $\mathrm{E}$ (K equals 0.352 and 0.372 , respectively) are considerably less than the average level. In the experiment, Child A and Child F's ratings for 
liking, anxiety, and engagement were almost constant which resulted in lower $\mathrm{K}$ values for the therapist and child pair $(\mathrm{T} / \mathrm{C})$ and the parent and child pair $(\mathrm{P} / \mathrm{C})$ than those of the other participants. This may be due to the fact that the spectrum developmental disorder for children with autism manifests different abilities to recognize and report their emotions. The mean of the kappa statistic values between the children and either the therapist or the parent were relatively small ( 0.37 and 0.40 , respectively). Although lack of agreement with adults does not necessarily mean that the self-reports of children with ASD are not dependable; however, given the fact that therapists' judgment based on their expertise is the state-of-the-art in most autism intervention approaches and the fact that there is a reasonably high agreement between the therapist and the parents for all of the six children, the subjective reports of the therapist were used as the reference points linking the objective physiological data to the children's affective state. To make the subjective reports more consistent, the same therapist was involved in all of the experiments. This choice allowed for building a therapist-like affective model. Once the affect modeling is completed, the recognizers will be capable of inferring the affective states of the child with ASD from the physiological signals in real-time even when the therapist is not available.

The performance of the developed affective model for each child (i.e., individual-specific approach) is shown in Fig. 8. The cross-validation method, 'leave-one-out', was used. The affective model produced high recognition accuracies for each target affective state of each participant. The average correct prediction accuracies across all participants were: $85.0 \%$ for liking, $79.5 \%$ for anxiety, and $84.3 \%$ for engagement, which are comparable to the best results achieved for typical individuals (Picard et al., 2001; Rani et al., 2006b). We also compared the performance of affective modeling to a control method that represents random chance. For example, in 48 out of 86 epochs the engagement of Child $E$ was rated as low level, where a random classification could assign all test epochs to this category and make accurate classifications $(48 / 86) \times 100=55.8 \%$ of the time. We thus considered the level with a majority of epochs to represent the chance condition, which is denoted by dark grey bars in Fig. 8. While the physiology-based affective modeling alone did not provide perfect classification (i.e., 100\%) of affective states of children with ASD, they did yield reliable matches with the subjective rating and significantly outperformed a random classifier (averaging $82.9 \%$ vs. $59.2 \%$ ). This was promising considering that this task was challenging in two respects: (i) the reports were collected from the therapist who was observing the children as opposed to having typical adults capable of differentiating and reporting their own affective states and (ii) varying levels of arousal of any given affective state (e.g., low/high anxiety) were identified instead of determining discrete emotions (e.g., anger, joy, sadness, etc.).

To explore the effects of reducing the number of physiological signals and the possibility of achieving more economical modeling, we examined the performance of the affect recognizers when cardiovascular, electrodermal, and electromyographic activities and their combinations were used. As shown in Table 3, all the recognizers delivered better predication than random guess (mean prediction rate equals $52.9 \%$ ), and with more information from physiological activities the performance of the affective models tends to improve (except the combination of electrodermal and electromyographic activities). While no combination of physiological activity surpassed the percent accuracy achieved when all signals were used, the results suggested it may be possible to selectively reduce the set of 
signals and obtain nearly-as-good performance (e.g., using a combination of cardiovascular and electrodermal signals).

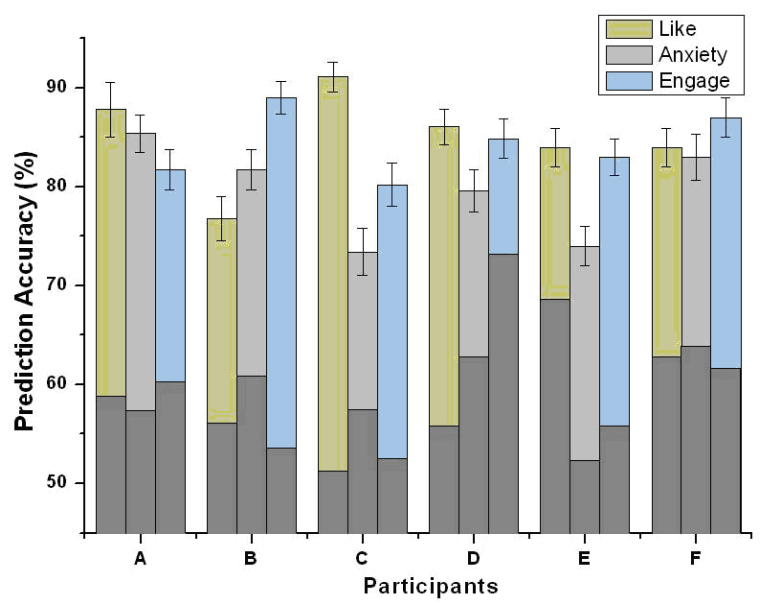

Fig. 8. Prediction Accuracy of the Affective Model

\begin{tabular}{|c|c|c|c|c|}
\hline Physiological Signals & Liking & Anxiety & Engage & Mean \\
\hline \hline Cardiovascular & 75.7 & 68.5 & 76.2 & 73.5 \\
\hline Electrodermal & 73.4 & 72.3 & 73.3 & 73.0 \\
\hline Electromyographic & 73.1 & 65.8 & 70.1 & 69.7 \\
\hline $\begin{array}{c}\text { Electrodermal + } \\
\text { Electromyographic }\end{array}$ & 75.0 & 69.4 & 71.4 & 71.9 \\
\hline $\begin{array}{c}\text { Cardiovascular + } \\
\text { Electromyographic }\end{array}$ & 79.6 & 70.2 & 79.9 & 76.6 \\
\hline Cardiovascular + Electrodermal & $\mathbf{7 9 . 9}$ & $\mathbf{7 4 . 3}$ & $\mathbf{8 1 . 9}$ & $\mathbf{7 8 . 7}$ \\
\hline All & $\mathbf{8 5 . 0}$ & $\mathbf{7 9 . 5}$ & $\mathbf{8 4 . 3}$ & $\mathbf{8 2 . 9}$ \\
\hline
\end{tabular}

Table 3. Prediction Accuracy of the Affective Modeling based on Different Physiological Signals $(\%)^{*}$

\subsection{Phase II - affect adaptation in robot-based basketball task}

Six children with ASD who completed the Phase I experiments also took part in the robot basketball task. The results described here are based on the RBB1 (non-affect based) and RBB2 (liking-based) tasks.

First, we present results to validate that different behaviors of the robot had distinguishable impacts on the liking level of the children with ASD. To reduce the bias of validation, in RBB1 the robot selects behaviors randomly and the occurrence of each behavior is evenly

${ }^{*}$ Peripheral temperature has relatively few features derived and was not examined independently. Instead, it was studied conjunctively with the electrodermal activity, both of which were acquired from the non-dominant hand of a participant. 
distributed. Fig. 9 shows the average labeled liking level for each behavior as reported by the therapist in RBB1. The difference of the impact is significant for five children (participants A, B, D, E, and F) and moderate for participant C. Across all participants, the differences of reported liking for the most-preferred, moderately-preferred, and leastpreferred behavior are statistically significant $(p<0.05$, ANOVA test). Furthermore, it was observed that different children with ASD may have different preferences for the robot's behaviors. These results demonstrated that it is important to have a robot learn the individual's preference and adapt to it automatically, which may allow a more tailored and affect-sensitive interaction between children with ASD and the robot.

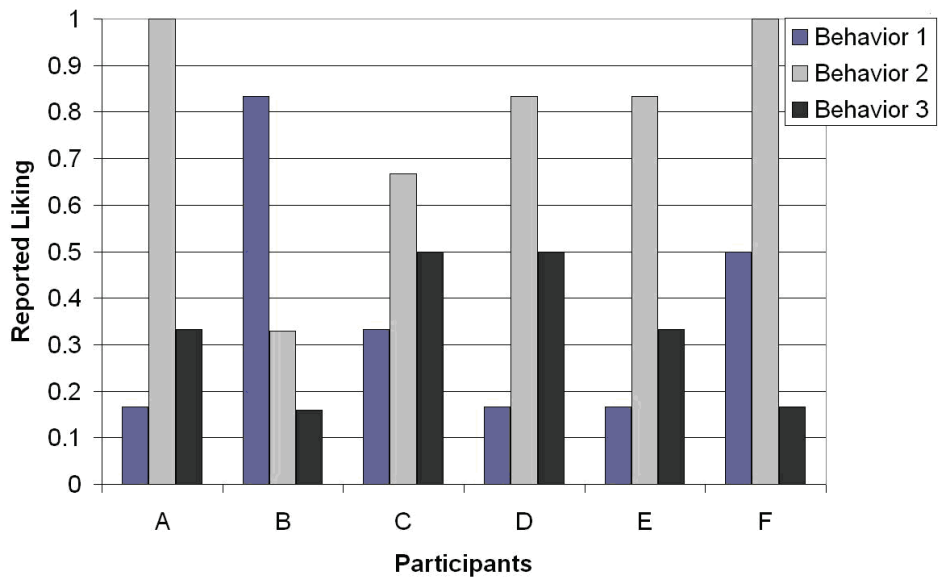

Fig. 9. Mean liking level for different behaviors in RBB1

Second, the predictive accuracy of how closely the real-time physiology-based quantitative measures of liking, as obtained from affective models developed in Phase I, matched with that of the subjective rating of liking made by the therapist during Phase II is discussed. The average predictive accuracy across all the participants was approximately $81.1 \%$. The highest was $86.1 \%$ for Child D, and the lowest was $77.8 \%$ for Child B and Child E. Note that the affective model was evaluated based on physiological data obtained on-line from a realtime application for children with ASD. However, this prediction accuracy is comparable to the results achieved through off-line analysis for typical individuals (Rani et al., 2006b).

Third, we present results about robot behavior adaptation and investigate its impact on the interaction between the children and the robot. Table 4 shows the percentages of different behaviors that were chosen in RBB2 for each participant. The robot learned the individual's preference and selected the most-preferred behavior with high probability for all the participants. Averaged across participants, the most-preferred, moderately-preferred, and least-preferred behaviors were chosen $72.5 \%, 16.7 \%$, and $10.8 \%$ of the time, respectively. The preference of a behavior was defined by the reported liking level in RBB1 as shown in Fig. 9. There could be several reasons why less-preferred behaviors were chosen in RBB2. The learned behavior selection policy might not have been optimal after the exploration in RBB1, and the QV-learning algorithm continued the learning process in RBB2. Another reason could be that the affective model is not $100 \%$ accurate and may return false reward/punishment, which may have given the robot imperfect instruction for behavior 
switches. Habituation to the most-preferred behavior during RBB2 could also be a factor that might have contributed to temporary changes in preference which led the robot to choose other behaviors.

\begin{tabular}{|c|c|c|c|c|c|c|}
\hline \multirow{2}{*}{ Child ID } & \multicolumn{2}{|c|}{$\begin{array}{c}\text { Most-Liked } \\
\text { Behavior }\end{array}$} & \multicolumn{2}{c|}{$\begin{array}{c}\text { Moderate-Liked } \\
\text { Behavior }\end{array}$} & \multicolumn{2}{|c|}{$\begin{array}{c}\text { Least-Liked } \\
\text { Behavior }\end{array}$} \\
\cline { 2 - 7 } & ID & Proportion & ID & Proportion & ID & Proportion \\
\hline A & 2 & $82.4 \%$ & 3 & $11.8 \%$ & 1 & $5.8 \%$ \\
\hline B & 1 & $70.6 \%$ & 2 & $17.7 \%$ & 3 & $11.7 \%$ \\
\hline C & 2 & $58.8 \%$ & 3 & $23.5 \%$ & 1 & $17.7 \%$ \\
\hline D & 2 & $76.5 \%$ & 3 & $11.8 \%$ & 1 & $11.7 \%$ \\
\hline E & 2 & $76.5 \%$ & 3 & $17.6 \%$ & 1 & $5.9 \%$ \\
\hline F & 2 & $70.6 \%$ & 1 & $17.7 \%$ & 3 & $11.7 \%$ \\
\hline
\end{tabular}

Table 4. Proportion of Different Behaviors Performed in RBB2

In Fig. 10 we present results to demonstrate that active monitoring of participants' liking and automatically selecting the preferred behavior allowed children with ASD to maintain high liking levels. The average labeled liking levels of the participants as reported by the therapist during the two sessions were compared. The lighter bars indicate the liking level during the RBB1 session (i.e., when the robot selected behaviors randomly), and the darker bars show the liking level during the RBB2 session (i.e., when robot learned the individual preference and chose the appropriate behavior accordingly). For all participants liking level was maintained, and for five of the six children liking level increased.

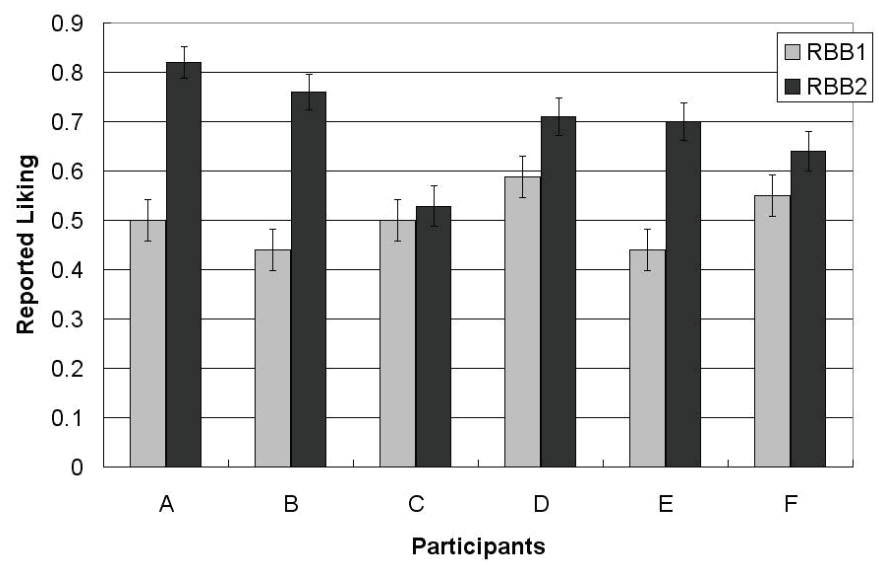

Fig. 10. Subjective liking as reported by therapist

There was no significant increase for Child $\mathrm{C}$ during the liking-based session as compared to the non-affect based session. The impact of the different robot behaviors on the liking level of Child C is not as significant as that of the others (refer to Fig. 9), which may impede the robot in finding the preferred behavior and hence impede the robot in effectively influencing the subjective liking level positively. Note that RBB1 presents a typically 
balanced interaction with equal numbers of most-preferred, moderately-preferred, and least-preferred epochs and the comparisons in Fig. 10 are not between liking-based sessions and sessions of least-preferred epochs. To determine whether this change in liking level was statistically significant across all the participants, a one-way ANOVA test was performed on the null hypothesis of no change in liking level between liking-based sessions and non-affect based sessions. The null hypothesis could be rejected at the $99.5 \%$ confidence level. This was a significant result as the robot continued learning and utilizing the information regarding the probable liking level of children with ASD to adjust its behaviors. This ability enables the robot to adapt its behavior selection in real time and hence keep the participant in a higher liking level.

\section{Conclusions and future work}

There is increasing consensus in the autism community that development of assistive tools that exploit advanced technology will likely make application of intensive intervention for children with ASD more readily accessible. In recent years, various applications of advanced interactive technologies have been investigated in order to facilitate and/or partially automate the existing behavioral intervention that addresses specific deficits associated with autism. However, the current technology-assisted intervention tools for children with ASD do not possess the ability of deciphering affective cues from the children, which could be critical given that the affective factors of children with ASD have significant impacts on the intervention practice. In this work, we have proposed a novel framework for affect-sensitive human-machine interaction where the intelligent system can detect the affective states of the children with ASD implicitly and respond to it accordingly.

The presented affective modeling methodology could allow the recognition of affective states of children with ASD from physiological signals in real time and provide the basis for future technology-assisted affect-sensitive interactive autism intervention. In Phase I, two cognitive tasks - solving anagrams and playing Pong - have been designed to elicit the affective states of liking, anxiety, and engagement for children with ASD that are considered important in autism intervention. To have reliable reference points to link the physiological data to the affective states, the reports from the child, the therapist, and the parent were collected and analyzed. A large set of physiological indices have been investigated to determine their correlation with the affective states of the children with ASD. We have experimentally demonstrated that it is viable to detect the affective states of children with ASD via a physiology-based affect recognition mechanism. A SVM-based affective model yielded reliable prediction with a success rate of $82.9 \%$ when using the therapist's reports.

In order to investigate the affect-sensitive closed-loop interaction between the children with ASD and an intelligent system, we designed a proof-of-concept task, robot-based basketball, and developed an experimental system for its real-time implementation and verification. The real-time prediction of liking level of the children with ASD was accomplished with an average accuracy of $81.1 \%$. The robot learned individual preferences of the children with ASD over time based on the interaction experience and the predicted liking level and hence automatically selected the most-preferred behavior, on average, $72.5 \%$ of the time. We have observed that such affect-sensitive robot behavior adaptation has led to an increase in reported liking level of the children with ASD. This is the first time, to our knowledge, that the affective states of children with ASD have been detected via a physiology-based affect recognition technique in real time. This is also the first time that the impact of affect- 
sensitive closed-loop interaction between a robot and children with ASD has been demonstrated experimentally.

The presented work requires physiological sensing that has its own limitations. For example, one needs to wear physiological sensors, and use of such sensors could be restrictive under certain circumstances. Given the rapid progress in physiological sensing clothing and accessories (Picard, 1997), we believe that physiology-based affect recognition can be appropriate and useful for the application of interactive autism intervention and could be used conjunctively with other modalities (e.g., visual and audio) to allow flexible and robust affective modeling for children with ASD. Moreover, none of the participants in this study had any objection to wearing the physiological sensors.

Future work will involve designing socially-directed interaction experiments that address the social communication deficits of children with ASD. We will investigate how to augment the interactive autism intervention by having an intelligent system (e.g., computer, VR environment, or robot) respond appropriately to the inferred affects based on the affective model described here. Specifically, we plan to integrate the real-time affect recognition and response system described in this research with a life-like android face developed by Hanson Robotics (www.hansonrobotics.com) and separately with an interactive virtual reality environment developed with Vizard software (www.worldviz.com). These intelligent systems can produce accurate examples of common facial expressions that convey affective states. This affective information could be used as feedback for empathy exercises to help children recognize their own emotions. Enhancements on the intervention process could also be envisioned. For instance, the intelligent system could exhibit interesting behaviors to retain the child's attention when it detects his/her liking level is low. Additionally, we will investigate fast and robust learning mechanisms that would permit an intelligent system's adaptive response in the more complex interaction tasks.

\section{Appendix}

\subsection{Pattern recognition using support vector machines}

SVM, pioneered by Vapnik (1998), is an excellent tool for classification (Burges, 1998). Its appeal lies in its strong association with statistical learning theory as it approximates the structural risk minimization principle. Good generalization performance can be achieved by maximizing the margin, where margin is defined as the sum of the distances of the hyperplane from the nearest data points of each of the two classes. SVM is a linear machine working in a high $k$-dimensional feature space formed by an implicit embedding of $n$ dimensional input data $X$ (e.g., a vector of derived physiology features as described in Section 3.2.4) into a $k$-dimensional feature space $(k>n)$ through the use of a nonlinear mapping $\phi(X)$. This allows for the use of linear algebra and geometry to separate the data, which is normally only separable with nonlinear rules in the input space. The problem of finding a linear classifier for given data points with known class labels can be described as finding a separating hyperplane $W^{T} \varphi(X)$ that satisfies:

$$
y_{i}\left(W^{T} \varphi\left(X_{i}\right)\right)=y_{i}\left(\sum_{j=1}^{k} w_{j} \phi_{j}\left(X_{i}\right)+w_{0}\right) \geq 1-\xi_{i}
$$

where $N$ represents the number of training data pairs $\left(X_{i}, y_{i}\right)$ indexed by $i=1,2, \ldots, N ; y_{i} \in\{+1$, -1 \} represents the class label (e.g., high/low intensity of a target affective state); $\varphi(X)=$ 
$\left[\phi_{0}(X), \phi_{1}(X), \ldots, \phi_{k}(X)\right]^{\mathrm{T}}$ is the mapped feature vector $\left(\phi_{0}(X)=1\right)$; and $W=\left[w_{0}, w_{1}, \ldots, w_{k}\right]$ is the weight vector of the network. The nonnegative slack variable $\xi_{i}$ generalizes the linear classifier with soft margin to deal with nonlinearly separable problems.

All operations in learning and testing modes are done in SVM using a so-called kernel function defined as $K\left(X_{i}, X\right)=\varphi^{T}\left(X_{i}\right) \varphi(X)$ (Vapnik, 1998). The kernel function allows for efficient computation of inner products directly in the feature space and circumvents the difficulty of specifying the non-linear mapping explicitly. The most distinctive fact about SVM is that the learning task is reduced to a dual quadratic programming problem by introducing the Lagrange multipliers $\alpha_{i}$ (Vapnik, 1998; Burges, 1998):

Maximize

$$
Q(\alpha)=\sum_{i=1}^{N} \alpha_{i}-\frac{1}{2} \sum_{i=1}^{N} \sum_{j=1}^{N} \alpha_{i} \alpha_{j} y_{i} y_{j} K\left(X_{i}, X_{j}\right)
$$

Subject to

$$
\sum_{i=1}^{N} \alpha_{i} y_{i}=0 \text { and } 0 \leq \alpha_{i} \leq C
$$

where $C$ is a user-defined regularization parameter that determines the balance between the complexity of the network characterized by the weight vector $W$ and the error of classification of data. The corresponding $\alpha_{i}$ multipliers are only non-zero for the support vectors (i.e., the training points nearest to the hyperplane), which induces solution sparseness. The SVM approach is able to deal with noisy data and over-fitting by allowing for some misclassifications on the training set (Burges, 1998). This characteristic makes it particularly suitable for affect recognition because the physiology data is noisy and the training set size is often small. Another important feature of SVM is that the quadratic programming leads in all cases to the global minimum of the cost function. With the kernel representation and soft margin mechanism, SVM provides an efficient technique that can tackle the difficult, high dimensional affect recognition problem.

\subsection{Behavior adaptation using QV-learning}

QV-learning (Wiering, 2005), a variant of the standard reinforcement learning algorithm Qlearning (Watkins and Dayan, 1992), was applied to achieve the affect-sensitive behavior adaptation. QV-learning keeps track of both a $Q$-function and a $V$-function. The $Q$-function represents the utility value $Q(s, a)$ for every possible pair of state $s$ and action $a$. The $V$ function indicates the utility value $V(s)$ for each state $s$. The state value $V\left(s_{t}\right)$ and Q-value $Q\left(s_{t}, a_{t}\right)$ at step $t$ are updated after each experience $\left(s_{t}, a_{t}, r_{t}, s_{t+1}\right)$ by:

$$
\begin{gathered}
V\left(s_{t}\right):=V\left(s_{t}\right)+\alpha\left(r_{t}+\gamma V\left(s_{t+1}\right)-V\left(s_{t}\right)\right) \\
Q\left(s_{t}, a_{t}\right):=Q\left(s_{t}, a_{t}\right)+\alpha\left(r_{t}+\gamma V\left(s_{t+1}\right)-Q\left(s_{t}, a_{t}\right)\right)
\end{gathered}
$$

where $r_{t}$ is the received reward that measures the desirability of the action $a_{t}$ when it is applied on state $s_{t}$ and causes the system to evolve to state $s_{t+1}$. The difference between (4) and the conventional Q-learning rule is that QV-learning uses $V$-values learned in (3) and is not defined solely in terms of $Q$-values. Since $V(s)$ is updated more often than $Q(s, a)$, QVlearning may permit a fast learning process (Wiering, 2005) and enable the intelligent system to efficiently find a behavior selection policy during interaction. 


\section{Acknowledgements}

The authors gratefully acknowledge the MARI (Marino Autism Research Institute) grant, the staff support from the Vanderbilt Treatment and Research Institute for Autism Spectrum Disorders for guidance during the development of experiments involving children with ASD, and the parents and children who participated in the presented research.

\section{References}

American Psychiatric Association. (2000). Diagnostic and statistical manual of mental disorders: DSM-IV-TR (4th ed.). American Psychiatric Association. Washington, DC

Asha, K., Ajay, K., Naznin, V., George, T., \& Peter, F. D. (2005). Gesture-based affective computing on motion capture data, Proceedings of the Int. Conf. on Affective Computing and Intelligent Interaction, Beijing, China

Barkley, R. A. (1998). Attention deficit hyperactivity disorder: A handbook for diagnosis and treatment $(2 \mathrm{ed}$.). Guilford Press. New York, NY

Bartlett, M. S., Littlewort, G., Fasel, I., \& Movellan, J. R. (2003). Real time face detection and facial expression recognition: development and applications to human computer interaction, Proceedings of the Computer Vision and Pattern Recognition Workshop, Madison, Wisconsin

Bee, H. \& Boyd, D. (2004). The Developing Child. (10th ed.). Pearson. Boston

Ben Shalom, D., Mostofsky, S. H., Hazlett, R. L., Goldberg, M. C., Landa, R. J., Faran, Y., McLeod, D. R., \& Hoehn-Saric, R. (2006). Normal physiological emotions but differences in expression of conscious feelings in children with high-functioning autism. J Autism Dev Disord, 36(3):395-400

Bernard-Opitz, V., Sriram, N., \& Nakhoda-Sapuan, S. (2001). Enhancing social problem solving in children with autism and normal children through computer-assisted instruction. J Autism Dev Disord, 31(4):377-384

Bradley, M. M. (2000). Emotion and motivation, In: Handbook of Psychophysiology, J. T. Cacioppo, L. G. Tassinary \& G. Berntson, (Eds.), 602-642, Cambridge University Press. New York, NY

Brown, R. M., Hall, L. R., Holtzer, R., Brown, S. L., \& Brown, N. L. (1997). Gender and video game performance. Sex Roles, 36(11-12):793 - 812

Burges, C. J. C. (1998). A tutorial on Support Vector Machines for pattern recognition. Data Mining and Knowledge Discovery, 2(2):121-167

Cacioppo, J.T., Berntson, G.G., Larsen, J.T., Poehlmann, K.M., \& Ito, T.A. (2000). The psychophysiology of emotion, In: Handbook of Emotions, Lewis, M., \& HavilandJones, J.M., (Eds.), The Guilford Press. New York, NY

Cowie, R., Douglas-Cowie, E., Tsapatsoulis, N., Votsis, G., Kollias, S., Fellenz, W., \& Taylor, J. (2001). Emotion recognition in human-computer interaction. IEEE Signal Processing Magazine, 18(1):32-80

Dautenhahn, K., \& Werry, I. (2004). Towards interactive robots in autism therapy: background, motivation and challenges. Pragmatics \& Cognition, 12(1):1-35

Dautenhahn, K., Werry, I., Salter, T., Boekhorst, R. T. (2003). Towards Adaptive Autonomous Robots in

Autism Therapy: Varieties of Interactions, IEEE International Symposium on Computational Intelligence in Robotics and Automation, Kobe 
Dawson, M. E., Schell, A. M., \& Filion, D. L. (1990). The Electrodermal System. In: Principles of Psychophysiology: Physical, Social, and Inferential Elements, Cacioppo, J.T., \& Tassinary, L.G., (Eds.), Cambridge University Press. Cambridge, MA

Dunn, L. M., \& Dunn, L. M. (1997). PPVT-III: Peabody Picture Vocabulary Test-Third Edition.American Guidance Service. Circle Pines, Minnesota

Ernsperger, L. (2003). Keys to Success for Teaching Students with Autism. Future Horizons

Fong, T., Nourbakhsh, I., \& Dautenhahn, K. (2003). A survey of socially interactive robots. Robotics and Autonomous Systems, 42(3-4):143-166

Gillott, A., Furniss, F., \& Walter, A. (2001). Anxiety in high-functioning children with autism. Autism, 5(3):277-286

Green, D., Baird, G., Barnett, A. L., Henderson, L., Huber, J., \& Henderson, S. E. (2002). The severity and nature of motor impairment in Asperger's syndrome: a comparison with Specific Developmental Disorder of Motor Function. Journal of Child Psychology and Psychiatry and Allied Disciplines, 43(5):655-668

Groden, J., Goodwin, M. S., Baron, M. G., Groden, G., Velicer, W. F., Lipsitt, L. P., Hofmann, S. G., \& Plummer, B. (2005). Assessing Cardiovascular Responses to Stressors in Individuals with Autism Spectrum Disorders. Focus on Autism and Other Developmental Disabilities, 20(4):244-252

Jacobson, J.W., Mulick, J. A., \& Green, G. (1998). Cost-benefit estimates for early intensive behavioral intervention for young children with autism - General model and single state case. Behavioral Interventions, 13:201-206

Kataoka, H., Kano, H., Yoshida, H., Saijo, A., Yasuda, M., \& Osumi, M. (1998). Development of a skin temperature measuring system for non-contact stress evaluation. IEEE Ann. Conf. Engineering Medicine Biology Society

Kleinsmith, A., Fushimi, T., \& Bianchi-Berthouze, N. (2005). An incremental and interactive affective posture recognition system, Proceedings of the UM 2005 Workshop: Adapting the Interaction Style to Affective Factors, Edinburgh, United Kingdom

Kozima, H., Nakagawa, C., \& Yasuda, Y. (2005). Interactive robots for communication-care: A case-study in autism therapy, Proceedings of the IEEE International Workshop on Robot and Human Interactive Communication, Nashville, Tennessee, August

Kulic, D., \& Croft, E. (2007). Physiological and subjective responses to articulated robot motion. Robotica, 25:13-27

Lacey, J. I., \& Lacey, B. C. (1958). Verification and extension of the principle of autonomic response-stereotypy. Am J Psychol, 71(1):50-73

Lee, C. M., \& Narayanan, S. S. (2005). Toward detecting emotions in spoken dialogs. IEEE Transactions on Speech and Audio Processing, 13(2):293-303

Liu, C., Rani, P., \& Sarkar, N. (2006). Human-Robot interaction using affective cues. Proceedings of the International Symposium on Robot and Human Interactive Communication, Hatfield, United Kingdom

Mandryk, R. L., \& Atkins, M. S. (2007). A fuzzy physiological approach for continuously modeling emotion during interaction with play technologies. International Journal of Human-Computer Studies, 65(4):329-347

Michaud, F. \& Theberge-Turmel, C. (2002). Mobile robotic toys and autism. In: Socially Intelligent Agents: Creating Relationships with Computers and Robots, K. Dautenhahn, A. H. Bond, L. Canamero, and B. Edmonds, (Eds.), 125-132, Kluwer Academic Publishers 
Moore, D. J., McGrath, P., \& Thorpe, J. (2000). Computer aided learning for people with autism - A framework for research and development. Innovations in Education and Training International, 37(3):218-228

Papillo, J.F., \& Shapiro, D., (1990). The cardiovascular system. In: Principles of Psychophysiology: Physical, Social, and Inferential Elements, Cacioppo, J.T., \& Tassinary, L.G., (Eds.), Cambridge University Press. Cambridge, MA

Pares, N., Masri, P., van Wolferen, G., \& Creed, C. (2005). Achieving dialogue with children with severe autism in an adaptive multisensory interaction: the "MEDIAte" project. IEEE Trans Vis Comput Graph, 11:734-743

Parsons, S., \& Mitchell, P. (2002). The potential of virtual reality in social skills training for people with autistic spectrum disorders. J Intellect Disabil Res, 46(Pt 5):430-443

Parsons, S., Mitchell, P., \& Leonard, A. (2005). Do adolescents with autistic spectrum disorders adhere to social conventions in virtual environments? Autism, 9(1):95-117

Pecchinenda, A., \& Smith, C. A. (1996). The affective significance of skin conductance activity during a difficult problem-solving task. Cogn. and Emotion, 10(5):481-504

Picard, R. W. (1997). Affective Computing. The MIT Press. Cambridge, MA

Picard, R. W., Vyzas, E., \& Healey, J. (2001). Toward machine emotional intelligence: Analysis of affective physiological state. IEEE Transactions on Pattern Analysis and Machine Intelligence, 23(10):1175-1191

Pioggia, G., Igliozzi, R., Ferro, M., Ahluwalia, A., Muratori, F., \& De Rossi, D. (2005). An android for enhancing social skills and emotion recognition in people with autism. IEEE Transactions on Neural Systems and Rehabilitation Engineering, 13(4):507-515.

Rani, P., Liu, C., \& Sarkar, N. (2006a). Affective feedback in closed loop human-robot interaction. Proceeding of the 1st ACM SIGCHI/SIGART Conference on Human-Robot interaction, Salt Lake City, Utah, USA, March

Rani, P., Liu, C. C., Sarkar, N., \& Vanman, E. (2006b). An empirical study of machine learning techniques for affect recognition in human-robot interaction. Pattern Analysis and Applications, 9(1):58-69

Rani, P., Sarkar, N., Smith, C. A., \& Kirby, L. D. (2004). Anxiety detecting robotic system towards implicit human-robot collaboration. Robotica, 22:85-95

Reeves, B., \& Nass, C. I. (1996). The media equation: how people treat computers, televisions, and new media as real people and places. Cambridge University Press. New York, NY

Robins, B., Dickerson, P., \& Dautenhahn, K. (2005). Robots as embodied beings Interactionally sensitive body movements in interactions among autistic children and a robot, Proceedings of the 14th IEEE International Workshop on Robot and Human Interactive Communication, Nashville, Tennessee, August

Robins, B., Dickerson, P., Stribling, P., \& Dautenhahn, K. (2004). Robot-mediated joint attention in children with autism: A case study in robot-human interaction. Interaction Studies, 5(2):161-198

Rogers, S. J. (1998). Empirically supported comprehensive treatments for young children with autism. J Clin Child Psychol, 27(2):168-179

Ruble, L. A., \& Robson, D. M. (2006). Individual and Environmental Determinants of Engagement in Autism. J Autism Dev Disord.

Rutter, M. (2006). Autism: its recognition, early diagnosis, and service implications. J Dev Behav Pediatr, 27(2 Suppl):S54-58 
Scassellati, B. (2005). Quantitative metrics of social response for autism diagnosis, Proc. IEEE International Workshop on Robot and Human Interactive Communication, Nashville, Tennessee, August

Schultz, R.T. (2005). Developmental deficits in social perception in autism: the role of the amygdala and fusiform face area. Int J Dev Neurosci, 23:125-41

Seip, J. A. (1996). Teaching the autistic and developmentally delayed: A guide for staff training and development. Delta. British Columbia

Siegel, S., \& Castellan, J. N. J. (1988). Nonparametric Statistics for the Behavioral Sciences. McGraw-Hill. New York, NY

Silver, M., \& Oakes, P. (2001). Evaluation of a new computer intervention to teach people with autism or Asperger syndrome to recognize and predict emotions in others. Autism, 5(3):299-316

Swettenham, J. (1996). Can children with autism be taught to understand false belief using computers? J Child Psychol Psychiatry, 37(2):157-165

Tarkan, L. (October 21, 2002). Autism therapy is called effective, but rare. New York Times

Toichi, M., \& Kamio, Y. (2003). Paradoxical autonomic response to mental tasks in autism. J Autism Dev Disord, 33(4):417-426

Trepagnier, C. Y., Sebrechts, M. M., Finkelmeyer, A., Stewart, W., Woodford, J., \& Coleman, M. (2006). Simulating social interaction to address deficits of autistic spectrum disorder in children. Cyberpsychol Behav, 9(2):213-217

Vansteelandt, K., Van Mechelen, I., \& Nezlek, J. B. (2005). The co-occurrence of emotions in daily life: A multilevel approach. Journal of Research in Personality, 39(3):325-335

Vapnik, V. N. (1998). Statistical Learning Theory. Wiley-Interscience. New York, NY

Watkins, C. J. C. H. \& Dayan, P. (1992). Q-Learning. Machine Learning, 8, May

Werry I, Dautenhahn K, \& Harwin W. (2001). Investigating a robot as a therapy partner for children with autism, Proceedings of the 6th European conference for the advancement of assistive technology, Ljubljana, Slovenia

Wieder, S., \& Greenspan, S. (2005). Can Children with Autism Master the Core Deficits and Become Empathetic, Creative, and Reflective? The Journal of Developmental and Learning Disorders, 9

Wiering, M. A. (2005). QV ( $\lambda$ )-learning: A New On-policy Reinforcement Learning Algorithm, European Workshop on Reinforcement Learning, Napoli, Italy, October 


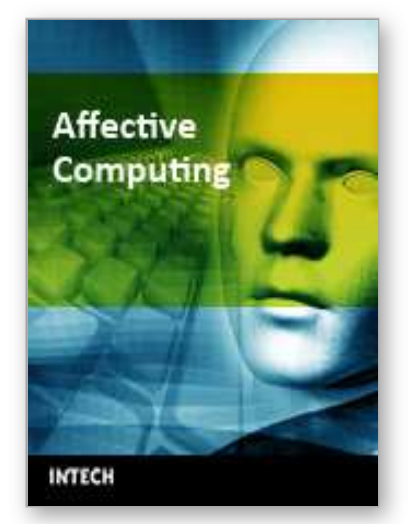

\author{
Affective Computing \\ Edited by Jimmy Or
}

ISBN 978-3-902613-23-3

Hard cover, 284 pages

Publisher I-Tech Education and Publishing

Published online 01, May, 2008

Published in print edition May, 2008

This book provides an overview of state of the art research in Affective Computing. It presents new ideas, original results and practical experiences in this increasingly important research field. The book consists of 23 chapters categorized into four sections. Since one of the most important means of human communication is facial expression, the first section of this book (Chapters 1 to 7) presents a research on synthesis and recognition of facial expressions. Given that we not only use the face but also body movements to express ourselves, in the second section (Chapters 8 to 11 ) we present a research on perception and generation of emotional expressions by using full-body motions. The third section of the book (Chapters 12 to 16) presents computational models on emotion, as well as findings from neuroscience research. In the last section of the book (Chapters 17 to 22) we present applications related to affective computing.

\title{
How to reference
}

In order to correctly reference this scholarly work, feel free to copy and paste the following:

Karla Conn, Changchun Liu, Nilanjan Sarkar, Wendy Stone and Zachary Warren (2008). Towards Affectsensitive Assistive Intervention Technologies for Children with Autism, Affective Computing, Jimmy Or (Ed.), ISBN: 978-3-902613-23-3, InTech, Available from:

http://www.intechopen.com/books/affective_computing/towards_affect-

sensitive_assistive_intervention_technologies_for_children_with_autism

\section{INTECH}

open science | open minds

\section{InTech Europe}

University Campus STeP Ri

Slavka Krautzeka 83/A

51000 Rijeka, Croatia

Phone: +385 (51) 770447

Fax: +385 (51) 686166

www.intechopen.com

\section{InTech China}

Unit 405, Office Block, Hotel Equatorial Shanghai

No.65, Yan An Road (West), Shanghai, 200040, China

中国上海市延安西路65号上海国际贵都大饭店办公楼405单元

Phone: +86-21-62489820

Fax: +86-21-62489821 
(C) 2008 The Author(s). Licensee IntechOpen. This chapter is distributed under the terms of the Creative Commons Attribution-NonCommercialShareAlike-3.0 License, which permits use, distribution and reproduction for non-commercial purposes, provided the original is properly cited and derivative works building on this content are distributed under the same license. 